Editorial

\title{
Cardiac rehabilitation after acute myocardial infarction: Still insufficient referral!... is
}

\author{
Ana Abreu \\ Hospital Santa Marta, CHLC, Cardiology Service, Rua Santa Marta, Lisboa, Portugal
}

\section{A R T I C L E I N F O}

\section{Article history:}

Received 20 February 2018

Accepted 9 March 2018

Cardiac Rehabilitation (CR) after acute myocardial infarction (AMI) is a class I recommendation in European and American guidelines, due to multiple clinical benefits and cost-effectiveness [1,2]. The positive prognostic value of $C R$ was recently reinforced by the CROS study [3] and the Cochrane meta-analysis [4], which unequivocally demonstrated, in the $\mathrm{PCI}$ era, a reduction in total and cardiac mortality in patients with acute coronary syndrome (ACS).

Regrettably, patients referred to $\mathrm{CR}$ in clinical practice are approximately half of those who are eligible [5]. Obstacles to referral, uptake and adherence are still a matter of concern. Different barriers are present, related to patients, doctors and healthcare-system. Effective strategies to increase uptake include, not only patient education and empowerment, but also post-hospital discharge specific sessions, structured follow-up, and planned early appointments to programs. In-hospital CR, phase 1 , the earliest noninvasive intervention during hospital stay after the acute event, should be a priority [6].

"Referral for cardiac rehabilitation after acute myocardial infarction: insights from a nationwide AMIS Plus registry 2005-2017" [7] aimed to evaluate the characteristics of patients referred to CR after AMI and to identify predictors of non-referral, using the large data of a prospective registry of 32,416 ACS patients admitted in Swiss hospitals. This study might give us helpful indications regarding this important issue, though conclusions cannot be extrapolated to other countries or groups of patients, which might have other barriers and non referral characteristics.

In 23,222 swiss ACS patients, with available orientation data at discharge, referral to CR occurred in $47.1 \%$ [7]. If we consider that not all the referred patients perform $\mathrm{CR}$, and some drop-out before terminating the program, the final number will be even lower. Based on the CR referral and uptake data of Euroaspire IV [5], we could say that $38,5 \%$ attended $C R$ programs.

\footnotetext{
th Regarding the Article: Referral for cardiac rehabilitation after acute myocardia infarction: insights from a nationwide AMIS Plus registry 2005-2017. Hermann M, Witassek F, Erne P, et al.

DOI of original article: https://doi.org/10.1016/j.ijcard.2018.01.096.

E-mail address: ananabreu@hotmail.com.
}

The referred patients were younger, more obese, with more STEMI and PCI. They also had more in-hospital complications, NYHA class III or IV, depressed left ventricular systolic function (LVEF $<30-35 \%$ ) and smoking habit. Age $>65$ years and presence of comorbidities, like peripheral artery disease, cerebrovascular disease and previous myocardial infarction, were negative determinants for CR [7].

Regarding to age and co-morbidities, we know, from previous studies, that older cardiac patients, frequently frail, with multi-morbidities, and those with co-morbidities, independently of age, are usually excluded from CR programs, so this result was expected. However, functional and psychological benefits, as better risk factors control, have been well documented in these patients [8]. Mobility, independence, social integration, psychological equilibrium and quality of life are important targets in this subset of patients, who might benefit the most in daily-life activities. Strategies to include patients with comorbidities and/or older, need to be developed, probably including home-based $\mathrm{CR}$ with long-distance monitoring techniques, also pursuing education and coaching.

More patients with significant heart failure, worst functional clinical class and left ventricular ejection fraction, were referred to CR [7]. Contrarily, most studies report less $\mathrm{CR}$ referral in heart failure, although these patients require, and obtain, the greater benefit from multidisciplinary approach and should begin CR soon after stabilization [8].

It was interesting to find out that more obese patients were referred to cardiac rehabilitation [7]. Probably doctors found difficult and timeconsuming trying by themselves to get the patients thinner. They understand that CR is a way of helping effective lifestyle modification, by diet, exercise, nutritional and psychological support which, with multidisciplinary team intervention, is unquestionably easier. Also, smoking is a difficult risk factor to manage, and the fact that patients submitted to CR were more frequently smokers, tell us that doctors understand the difficulty to interrupt this habit. Specific teams with smoking cessation techniques need to be present in CR for this difficult lifestyle change.

Patients with STEMI, the considered "major" infarction, mostly treated with PCI [7], were probably more frightening and subject to attention, as well as those with in-hospital complications, like cardiogenic shock, re-infarction, bleeding and cerebrovascular event. The aim of CR, in those without complications, is to recover, not functionally because hospital stay is getting much shorter with less immobilization problems, but psychologically, targeting orientation to work, to travel, to return to usual life including sexual activity and especially to improve prognosis with risk factors control, pharmachologic therapy management and adherence promotion. One of the big post-AMI problems is drug therapy 
non-adherence, which increases mortality and morbidity [9]. In this study [7], PCI patients were more referred to CR probably because of the fear of stent occlusion due to anti-platelet drugs suspension. In those patients with complications, an enlarged recovery after prolonged hospitalization, including the important functional component of rehabilitation and physiologic equilibrium management is crucial.

Patients with NSTEMI are usually older and present more comorbidities, but most of the times are considered less severe than STEMI. Consequently, they are less referred and adhere less to CR. This doctor's misconception must be corrected, because NSTEMI compared to STEMI patients, though at lower risk at the time of infarction, have similar or even worse prognosis, in the long term [10].

A good strategy for CR is to convince doctors to include all ACS in phase 1 CR [6]. The admission to in-hospital phase, if possible when the patient is still in ICU, will increase the number of patients referred to ambulatory phase $2 \mathrm{CR}$. Educational sessions and good communication with health providers, doctors, nurses and nutritionists, discussing risk factors and healthy lifestyle, besides socio-psychological support and functional training onset, will provide, while still in the hospital, more than recovery, confidence and motivation for continuing.

Studies like this, performed in Switzerland [7], should be replicated in more countries, who undertake ACS registries, including the referral to $\mathrm{CR}$ at discharge. The ideal, would be to cross these data with CR programs registries. It would allow us to understand each reality in referral and uptake obstacles and, consequently, to define and tailor strategies to increase and improve CR in all subsets of ACS patients. Nevertheless, independently of all studies on patients' characteristics, doctors need to believe that through $\mathrm{CR}$ referral they are offering their patients the best intervention after AMI and PCI.

\section{Conflict of interest}

I have no conflict of interest to declare.

\section{References}

[1] P. Giannuzzi, H. Saner, H. Bjornstad, et al., Secondary prevention through cardiac rehabilitation: position paper of the working group on cardiac rehabilitation and exercise physiology of the European society of cardiology, Eur. Heart J. 24 (2003) 1273-1278.

[2] E.M. Antman, S.T. Anbe, P.W. Armstrong, et al., ACC/AHA guidelines for the management of patients with ST-elevation myocardial infarction: executive summary: a report of the American College of Cardiology/American Heart Association Task Force on Practice Guidelines, J. Am. Coll. Cardiol. 44 (2004) 671-719.

[3] B. Rauch, C. Davos, P. Doherty, et al., The prognostic effect of cardiac rehabilitation in the era of acute revascularisation and statin therapy: a systematic review and metaanalysis of randomized and non-randomized studies - the cardiac rehabilitation outcome study (CROS), Eur. J. Prev. Cardiol. 23 (18) (2016) 1914-1939.

[4] L. Anderson, N. Oldridge, D.R. Thompson, et al., Exercise based cardiac rehabilitation for coronary heart disease, J. Am. Coll. Cardiol. 67 (1) (2016) 1-12.

[5] K. Kotseva, D. Wood, D. De Bacquer, et al., Euroaspire IV: a European society of cardiology survey on lifestyle, risk factor, and therapeutic management of coronary patients from 24 European countries, Eur. J. Prev. Cardiol. 23 (2016) 636-648.

[6] M. Piepoli, U. Corrà, P. Dendale, et al., Challenges in secondary prevention after acute myocardial infarction: a call for action, Eur. J. Prev. Cardiol. 23 (18) (2016) 1994-2006.

[7] M. Hermann, F. Witassek, P. Erne, et al., Referral for cardiac rehabilitation after acute myocardial infarction: insights from nationwide AMIS Plus Registry 2005-2017, Int. J. Cardiol. 261 (2018) 1-5.

[8] M. Piepoli, U. Corrà, W. Benzer, et al., Secondary prevention through cardiac rehabilitation:from knowledge to implementation. A position paper from the cardiac rehabilitation section of the European association of cardiovascular prevention and rehabilitation, Eur. J. Prev. Cardiol. 17 (2010) 1-17.

[9] P.M. Ho, J.A. Spertus, F.A. Masoudi, et al., Impact of medication therapy discontinuation on mortality after myocardial infarction, Arch. Intern. Med. 166 (2006) 1842-1847.

[10] C.J. Terkelsen, J.F. Lassen, B.L. Norgaard, et al., Mortality rates in patients with STelevation vs. non-ST-elevation acute myocardial infarction: observations from an unselected cohort, Eur. Heart J. 26 (2005) 18-26. 